\title{
Radial distribution of planets
}

\section{Predictions based on the core-accretion gas-capture planet-formation model}

\author{
K. Kornet ${ }^{1,2}$ and S. Wolf ${ }^{1}$ \\ 1 Max-Planck-Institute for Astronomy, Königstuhl 17, 69117 Heidelberg, Germany \\ e-mail: kornet@mpia.de; swolf@mpia.de \\ 2 Nicolaus Copernicus Astronomical Center, Bartycka 18, Warsaw 00-716, Poland
}

Received 24 July 2005 / Accepted 2 January 2006

ABSTRACT

\begin{abstract}
We have investigated the problem of the distribution of both masses and orbital radii of planets resulting from the gas-accretion, gas-capture model. First we followed the evolution of gas and solids from the moment where all solids are in the form of small grains to the stage when most of them are in the form of planetesimals for a set of different initial masses and sizes of protoplanetary disks. Based on that we performed Monte-Carlo calculations describing the formation of giant places at different locations. We included the effects of type II migration and growth of the mass of the planet after the gap opened. We discuss how these effects influence the final distribution of giant planets. We show that when the giant planets are not able to migrate or grow in mass after the gap opens, their distribution is mainly determined by the properties of the gaseous disk. However, with those two effects included, reproducing the parameters of the gaseous disks from the distribution of planets becomes difficult. We also checked the roles of both the material of which the solids consist and the mass of the central star. The main result is that, in disks around less massive stars, giant planets at the given location tend to be less massive. At the same time, the giant planets with the given mass tend to form closer to the less massive stars.
\end{abstract}

Key words. planetary systems: formation - planetary systems: photoplanetary disks

\section{Introduction}

Current radial velocity surveys have led to the discovery of over 150 extrasolar planets around main-sequence stars (see Marcy et al. 2005; Mayor et al. 2004). The large majority of these planets are probably gas giant planets, as their masses are above $100 M_{\oplus}$. Such a collection provides a good data set for comparing predictions of theories of planet formation (e.g. Ida \& Lin 2004a,b; Alibert et al. 2005; Kornet et al. 2005).

The standard model for the formation of giant planets is the core-accretion, gas capture-model. The numerical calculations of Pollack et al. (1996) show, that the formation of a giant planet in this model can be divided into three phases. During the first one, the solid core is formed by the collisional accumulation of planetesimals. The second phase begins when the core reaches the mass of a few Earth masses and starts to accrete a significant amount of gas. During this phase the envelope stays in quasistatic and thermal equilibrium, as the energy radiated by the envelope is compensated for by the energy released by accreted planetesimals. As during this phase the protoplanet accretes gas with a higher rate than solids, the mass of the envelope finally becomes equal to the mass of the core. At this moment phase 3 begins, during which the planet rapidly grows in mass by runaway accretion of gas. The final mass and location of a giant planet are determined by its gravitational interaction with its environment. As it grows in mass it induces spiral waves in the gaseous disk. This leads to the transfer of angular momentum resulting in the inward migration of the planet and possibly in the gap opening (Lin \& Papaloizou 1986; Lin et al. 1996; Ward 1997). This last phenomenon strongly reduces the further growth of the planet.
The main problem with that scenario is related to the timescale required to form a giant planet in it. In general it is the same order of magnitude as the lifetime of the protoplanetary disk, and it is not necessarily certain if the giant planet is able to form before dispersion of the disk. Close to the star, the formation time of a giant planet in the gas capture model is determined by phase 2 , while at larger distances ( $\gtrsim 10 \mathrm{AU})$ the lengths of phases 1 and 2 become comparable. The lengths of these two phases depend on the initial surface density of the planetesimal swarm in the given location. The larger the density, the faster the core grows and reaches higher mass at the end of phase 1 . With the higher mass of the core, the length of phase 2 also diminishes. In general, at every distance from the star there is a critical value of the surface density of planetesimals which enables formation of a giant planet before dispersion of the protoplanetary disk; for a more detailed discussion see Kornet et al. (2006).

However, the density of the protoplanetary swarm is not in a simple relation with the density of gas in the disk from which it emerges. While the gaseous component evolves in a viscous way, the evolution of the solid component is governed by processes of gas drag, coagulation, sedimentation, and evaporation (Weidenschilling \& Cuzzi 1993). A significant redistribution of solid material takes place in the result, and in the inner parts of the disk its surface density can be significantly enhanced compared to the initial value (Stepinski \& Valageas 1997; Weidenschilling 2003). Consequently, analysis of the possible masses and orbits of giant planets resulting from the core accretion scenario should also include the global evolution of solids in protoplanetary disks. A simple model of this evolution was proposed by Kornet et al. (2001) and further extended by 
Kornet et al. (2002) and Kornet et al. (2005) to include subsequent formation of giant planets.

In this paper we extend our models to also include the effects of migration and the gap opening by the planet. It enables us for the first time to characterize the distribution of planetary masses and final locations resulting from the core accretion model, not only including the growth of the planet but also the preceding evolution of solids, which determines the surface density of the planetesimal swarm. In Sect. 2 we explain our approach to the evolution of the protoplanetary disk and planet formation. The results are presented in Sect. 3 and discussed in Sect. 4.

\section{Methods}

In our approach we divide the formation of giant planets into three phases in a natural way. In the first one the planetesimal swarm is formed in the protoplanetary disk by the collisional accumulations of solids. This phase lasts till the gravitational interactions between solid bodies become the dominant factor governing their evolution. In the second epoch the giant planet is build by the accretion of the planetesimals, and at later stages of gas onto the protoplanetary core. Finally, when the mass of the planet reaches a critical value, the planet migrates within the disk and reaches its final position. We now discuss how we modeled each of these phases.

\subsection{Formation of a planetesimal swarm}

We describe the protoplanetary disk as a two-component fluid consisting of gas and solids. We modeled the gaseous component as a geometrically thin turbulent $\alpha$ disk (Shakura \& Sunyaev 1973). Its surface density $\Sigma$ is given as a function of distance $a$ from the star and time $t$ in terms of a selfsimilar solution of Stepinski (1998). All other quantities characterizing the gas were obtained by solving the standard set of equations for a thin-disk approximation (e.g. Frank et al. 1992). The initial conditions were parameterized by two quantities: the initial mass of the disk $M_{0}$ and its initial outer radius $R_{0}$.

The crucial approximation underlying our approach to the evolution of solids is that the size distribution of particles at any given radial location is narrowly peaked around a mean value particular to this location and time. In the practical implementations it means that sizes of the particles $s$ are expressed as a single-value function of time and position, $s=s(t, r)$. Collectively, all solid particles are treated as a turbulent fluid characterized by its mean surface density $\Sigma_{\mathrm{s}}(t, r)$, which is governed by the continuity equation:

$\frac{\partial \Sigma_{\mathrm{s}}}{\partial t}+\frac{1}{r} \frac{\partial}{\partial r}\left(r V_{\mathrm{s}} \Sigma_{\mathrm{s}}\right)=0$.

The radial drift velocity of solids $V_{\mathrm{s}}$ is the result of the frictional coupling between solids and the gas and depends on the local size of the particles and properties of the gas. In the regions where the temperature of the disk is higher than the evaporation temperature $T_{\text {evap }}$, the solids are treated as vapour with a velocity equal to the velocity of the gas.

The size of the particles is governed by the second equation:

$\frac{\partial \Sigma_{\text {size }}}{\partial t}+\frac{1}{r} \frac{\partial}{\partial r}\left(r V_{\mathrm{s}} \Sigma_{\text {size }}\right)=f \Sigma_{\mathrm{s}}$

where $\Sigma_{\text {size }}=s \Sigma_{\mathrm{s}}$. The source function $f$ describes the growth of particles due to mutual collisions and subsequent coagulation.
The main assumptions used in its derivation are that all collisions between particles lead to coagulation and that the relative velocities of colliding particles are given by the turbulent model described by Stepinski \& Valageas (1997). In the calculation of the density of solids from their surface density, the effect of their sedimentation toward the midplane of the disk is taken into account by evolving the scale height of the solid disk.

Initially the surface density of solids is everywhere a constant fraction of the surface density of gas, and their sizes amount to $10^{-3} \mathrm{~cm}$. The results discussed in the subsequent sections do not depend on the choice of that particular value, as long as the solids are initially small enough to couple well to the gas.

\subsection{Growth of planets}

Our procedure for the formation of the giant planet starts when the planetesimals at a given point in the disk reach radii of $2 \mathrm{~km}$. That moment determines the initial surface density of the planetesimal swarm. The growth of the protoplanetary core is described by the following formula given by Papaloizou \& Terquem (1999):

$\dot{M}_{\mathrm{c}}=C_{1} C_{\text {cap }} R_{\mathrm{p}} R_{\mathrm{H}} \Omega_{\mathrm{K}} \Sigma_{\mathrm{s}}$

where

$R_{\mathrm{H}}=a\left(\frac{M_{\mathrm{p}}}{3 M_{\star}}\right)^{1 / 3}$

is the radius of the Hill sphere of the planet and $\Omega_{\mathrm{K}}$ the Keplerian angular velocity. The value of $C_{1}$ given by Papaloizou $\&$ Terquem (1999) is $81 \pi / 32$; we use a factor of 5 (the difference comes from the different definition of $R_{\mathrm{H}}$ ). The quantity $C_{\text {cap }}$ describes the increase in the effective capture radius of the planet within respect to its real radius $R_{\mathrm{p}}$ due to the interaction of planetesimals with the envelope of the planet (Podolak et al. 1988). We use for it an approximate fit provided by Hubickyj (2001), made to the results of Bodenheimer et al. (2000). For those core masses that are less then $5 M_{\oplus}$, no increase in the effective capture radius is assumed, i.e. $C_{\text {cap }}\left(M_{\mathrm{c}}<5 M_{\oplus}\right)=1$. For larger core masses, it increases linearly with the mass of the core, reaching its maximum value of $C_{\text {cap }}=5$ for $M_{\mathrm{c}}=15 M_{\oplus}$. We assume that the surface density of planetesimals $\Sigma_{\mathrm{s}}$ is constant throughout the feeding zone which, extends to 4 Hill radii on both sides of the planetary orbit. Furthermore, $\Sigma_{\mathrm{s}}$ changes only due to accretion of the planetesimals onto the core and the growth of the feeding zone.

Significant accretion of gas onto the core begins when it reaches a critical mass of

$M_{\mathrm{c}, \mathrm{crit}}=10\left(\frac{\dot{M}_{\mathrm{c}}}{10^{-6} M_{\oplus} \mathrm{yr}^{-1}}\right)^{0.25}\left(\frac{\kappa_{\mathrm{env}}}{1 \mathrm{~cm}^{2} \mathrm{~g}^{-1}}\right)^{0.25}$

(Ikoma et al. 2000), where $\kappa_{\text {env }}$ is the opacity in the envelope of the planet. Its actual magnitude is currently poorly constrained. We assume that $\kappa_{\text {env }}=1 \mathrm{~cm}^{2} \mathrm{~g}^{-1}$. When the mass of the protoplanet exceeds $M_{\mathrm{c}, \mathrm{crit}}$, it contracts on the Kelvin-Helmholtz time scale $\tau_{\mathrm{KH}}$. By fitting the result of Pollack et al. (1996) Bryden et al. (2000) show that

$\tau_{\mathrm{KH}}=10^{b}\left(\frac{M_{\mathrm{p}}}{M_{\oplus}}\right)^{-c}\left(\frac{\kappa}{1 \mathrm{~cm}^{2} \mathrm{~g}^{-1}}\right) \mathrm{yr}$

where the exponents $b=10$ and $c=3$. However, the gas accretion rate connected with this contraction cannot be higher than the speed with which viscous transport of gas replenishes the 
feeding zone of the planet. Consequently, we adopt the following equations for the gas accretion rate onto the star

$\frac{\mathrm{d} M_{\mathrm{env}}}{\mathrm{d} t}=\min \left[\frac{M_{\mathrm{p}}}{\tau_{\mathrm{KH}}}, \dot{M}_{\mathrm{disk}}\right]$

where $\dot{M}_{\text {disk }}$ is the accretion rate in the disk without the planet. We assume that it is equal to $\pi \alpha C_{\mathrm{s}} H \Sigma$, where $C_{\mathrm{S}}$ is the sound speed in the gaseous disk, $H$ its scale height, and $\Sigma$ its gas surface density at the given location.

When the Hill radius of the planet becomes larger than 1.5 of the scale height of the disk, the planet induces a strong tidal torque on the disk and opens a gap in it. As a result the accretion of gas is strongly diminished. Its maximum rate is then equal to:

$\dot{M}_{\text {env }}=\dot{M}_{\text {disk }}\left\{1.668\left(\frac{M_{\mathrm{p}}}{M_{\mathrm{J}}}\right)^{1 / 3} \exp \left[-\frac{M_{\mathrm{p}}}{1.5 M_{\mathrm{J}}}\right]+0.04\right\}$

where $M_{\mathrm{J}}$ is the Jupiter mass (Veras \& Armitage 2004).

\subsection{Migration}

The gravitational interaction of a planet with the disk leads to its migration and to formation of the gap in the disk (Lin \& Papaloizou 1986; Ward 1997). For a low-mass planet the interaction is linear and results in so-called type I migration. In contrast to this scenario, high-mass planets open a gap in the disk that reduces the timescale of migration (referred to as type II migration).

Analytical calculations show that the timescale of type I migration in a laminar disk is much shorter than both the disk lifetime and the timescale of planet formation. Consequently, if type I migration has taken place, nearly all planets would be accreted onto the star and the probability of finding any planetary system would be very low. Likewise, recent simulations by Nelson \& Papaloizou (2004) suggest that the torques exerted onto a low mass planet in a turbulent MHD disk fluctuate strongly. As a result the planet proceeds at a random walk and the mean value of the migration velocity is highly reduced. Due to these uncertainties we neglect the type I migration in our models.

Type II migration begins when the planet is massive enough to open a gap in the gas. It happens when the Hill radius of the planet is larger than 1.5 of the disk scale height. If the mass of the planet is negligible in comparison to the mass of the disk, the inward velocity of the planet is regulated by the viscosity of the disk (Ward 1997):

$\frac{\mathrm{d} a}{\mathrm{~d} t}=-\alpha \frac{C_{\mathrm{s}}^{2}}{a \Omega_{\mathrm{k}}}$

where $C_{\mathrm{s}}$ is the sound velocity in the gas. In the case of a planet with a larger mass than the mass of the disk, the migration is slowed down. The variation in the planetary angular momentum is then equal to the angular momentum transport rate in the disk (Lin et al. 1996):

$\frac{\mathrm{d}}{\mathrm{d} t}\left[M_{\mathrm{p}} a^{2} \Omega_{\mathrm{k}}\right]=-\frac{3}{2} \nu \Sigma \Omega_{\mathrm{k}} a^{2}$

In our calculations we used the smaller of the values of $\mathrm{d} a / \mathrm{d} t$ given by Eqs. (9) and (10). The migration of the planet was calculated using the 4 th order Runge-Kutta method. It stops when either the time from the beginning of the calculation (including the time needed for the formation of the planetesimal swarm) is longer then the lifetime of the protoplanetary disk $\tau_{\mathrm{f}}$, or the orbit of the planet becomes smaller than 0.01 AU. In the latter case we assumed that the planet is accreted onto the star. In our calculations $\tau_{\mathrm{f}}=3 \times 10^{6} \mathrm{yr}$, which agrees with observations (Haisch et al. 2001).

\section{Results}

Using the methods described in the previous section we were able to investigate the range of masses and orbits of giant planets which are able to form in protoplanetary disks characterized by different initial conditions. First, we calculated a grid of models of protoplanetary disks. Every model is characterized by two parameters: the initial mass $\left(M_{0}\right)$ and outer radius $\left(R_{0}\right)$ of the gaseous disk. Their initial masses are uniformly distributed between 0.02 and $0.2 M_{\odot}$. The range of outer radii was chosen with the goal of including all models in which the formation of giant planets is possible. The number of radii is constant per interval of $\lg R_{0}$. The viscosity parameter $\alpha$ is equal to 0.001 for all models. The solids consist of water ice with a bulk density of $1 \mathrm{~g} \mathrm{~cm}^{-3}$ and have an evaporation temperature of $150 \mathrm{~K}$. For every value of $\left[M_{0}, R_{0}\right]$ we also checked the gravitational stability of the corresponding gaseous disk. In some cases the value of the Toomre parameter

$Q=\frac{C_{\mathrm{S}} \Omega_{\mathrm{K}}}{\pi G \Sigma}$

drops below 1 in the outer regions of the disk. It means that they are gravitationally unstable with respect to the axisymmetric modes. We assumed that these parts of the disk fragment and some giant planets are formed there on a very short time scale (see i.e. Boss 2002). Consequently we used modified initial values of the mass and outer radius of the disk, which correspond to the mass and size of the stable part of the original disk. Our distribution the of initial parameters of protoplanetary disks is an artificial one. In general one should use the distribution that occurs in nature. Unfortunately current observations do not provide such information. However, our results remain valid, as we are interested only in characterizing the general set of possible orbital sizes and masses of planets.

Using that grid of evolved models we performed MonteCarlo calculations to produce the $M_{\mathrm{p}}-a$ distribution of planets resulting from our models. With the uniform probability we randomly chose one of the models from the calculated grid. Next, we chose the initial distance of the planet from the star $a$ with constant probability per interval of $\lg a$. We evolved the mass and semimajor axis of the planet according to the equations given in Sects. 2.2 and 2.3, starting from the time at which the planetesimals at radial distance $a$ reach a radius of $2 \mathrm{~km}$ in the given model of the protoplanetary disk. To better illustrate how the type II migration and the accretion of gas after opening the gap change the parameters of the giant planets we also performed calculations in which those two phenomena were not included.

The results of calculations in which the planets were neither permitted to increase their masses after the gap opening in the disk or to change their semimajor axes are presented in the upper panel of Fig. 1. It shows that the planets can be divided into two main groups (see also Fig. 2). In the first one there are bodies with masses smaller than $\sim 20 M_{\oplus}$. These bodies were not able to accrete a significant amount of gas so the nearly only consist of their solid cores. In the second group, there are planets with masses larger than $\sim 100 M_{\oplus}$. In these cases the runaway gas accretion has already taken place and the majority of their masses 


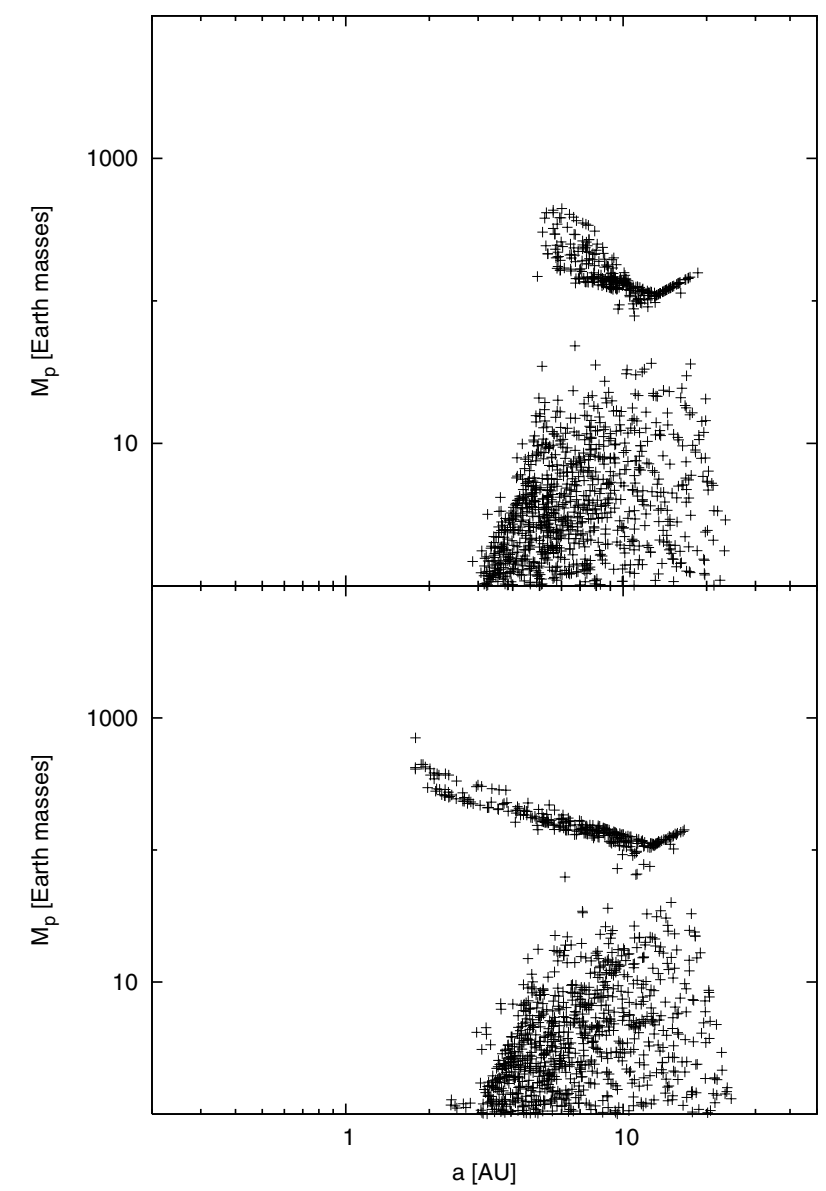

Fig. 1. The distribution of theoretically predicted semimajor axes and masses of planets around a star with a mass of $1 M_{\odot}$. The upper panel shows planets that neither migrate nor grow in mass after they are massive enough to open the gap in the disk. The lower one presents results for planets that undergo type II migration, but do not grow after gap opening. The solid material in the disk is water ice.

are in the form of gaseous envelopes. They are massive enough to open a gap in the disk. The small number of planets with intermediate masses results from the fact that, as the mass of the planet grows above $\sim 20 M_{\oplus}$, the timescale of the accretion of gas quickly becomes much shorter than the lifetime of the protoplanetary disk. Consequently, the probability of the disk to disperse before the planet grows sufficiently to open a gap is very low (see Ida \& Lin 2004a).

The giant planets in this set of models form in a region between 1 and $20 \mathrm{AU}$ from the central star. They can be divided into two subgroups, each of them forming one of the wings of the V-like shape of the distribution of giant planets on the $a-M_{\mathrm{p}}$ diagram. As the final mass of the giant planets in this set of models is determined by equation

$M_{\mathrm{p}}=3\left(\frac{1.5 H}{a}\right)^{1 / 3} M_{\star}$,

those two subgroups of planets reflect different ways in which the $H / a$ ratio depends on distance from the star $a$ at the moment of gap opening. Giant planets at distances larger than $\sim 10 \mathrm{AU}$ are at that time in the optically thin parts of the planetary disk in which $(H / a) \sim a^{7 / 20}$ (see Stepinski 1998). Consequently, the masses of these planets are correlated with their distances from the star. The correlation is extremely narrow because scale height of the disk does not depend on its initial mass and outer

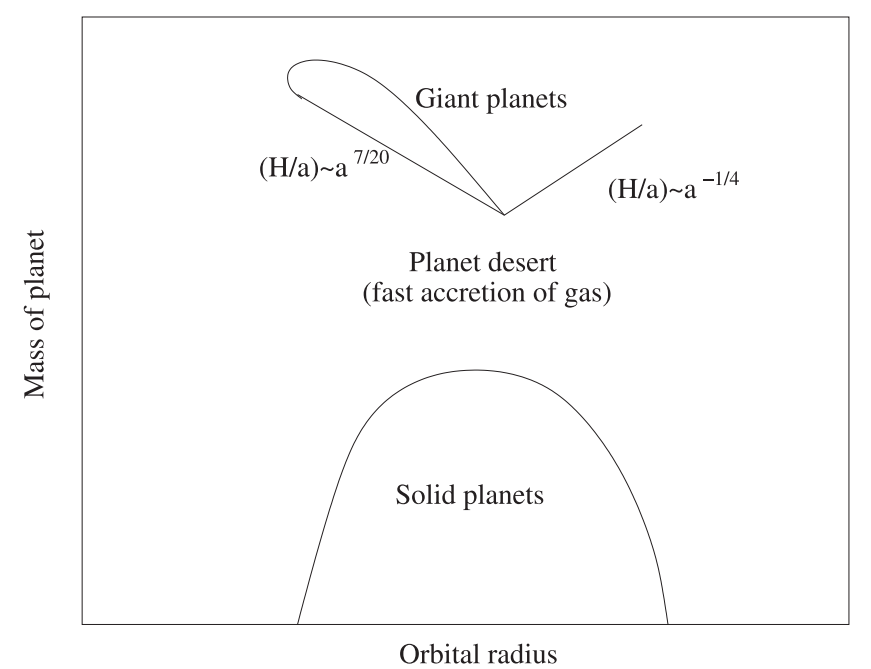

Fig. 2. The schematic diagram of distribution of theoretically predicted semimajor axes and masses of planets around a star with a mass of $1 M_{\odot}$ without effects of the gap opening and subsequent accretion of gas onto the planet.

radius in this regime in the used model of the gaseous disk. Giant planets at orbits smaller than $\sim 10 \mathrm{AU}$ in the moment of gap opening are in the optically thick part of the disk in which $(H / a) \sim a^{-1 / 4}$, so in their case their masses are anticorrelated with the distance from the star. The spread of this correlation is larger, because in that case the exact form of the $H(a)$ relation depends on the initial mass and radius of the underlying disk. This subgroup contains the most massive, closest planets formed in this set of simulations. They have masses around $450 M_{\oplus}$ and orbits around $4 \mathrm{AU}$.

In the next set of models we include the effects of type II migration, while planets still do not accrete any more after gap opening. The dependence of the masses of the planets resulting from this simulations on the sizes of their orbits is presented in the lower panel of Fig. 1. As expected, the only differences in comparison with the previous case are in the region of the giant planets. Moreover, the range of the masses of these planets in both figures are the same. Giant planets at larger orbits open the gap later because the process of their formation is longer due to lower densities of planetesimal swarm and smaller Keplerian frequencies (see Eq. (3)). Consequently the changes in their orbits are smaller as they have less time to migrate. Also the accretion rate in the disk that determines the speed of migration is lower at later times. In our calculations, only planets at orbits smaller than $\sim 9 \mathrm{AU}$ are able to shrink their orbits by a factor larger than $10 \%$. As the result, the distribution of planets on the $a-M_{\mathrm{p}}$ diagram for $a>9 \mathrm{AU}$ is nearly the same as in the case without migration. In the case of planets formed at smaller distances from the star, some of them are able to migrate to orbits as small as $\sim 2$ AU. Additionally, more massive planets tend to shrink their orbits by a larger factor. This can be explained by the higher rate of migration in the hotter disks. At the same time such disks have larger scale heights, and giant planets can reach larger masses before gap opening and stopping their growth. All these factors lead to the small spread of giant planets around a curve on the $a-M_{\mathrm{p}}$ diagram.

In the third set of calculations, the planets both migrate and grow in mass after gap opening. The distribution of the resulting planets is shown in Fig. 3. The range of their final semimajor axes is similar to the one in the previous set of models. The maximum size of planets at the given distance is $\sim 2000 M_{\oplus}$ at $2 \mathrm{AU}$ 


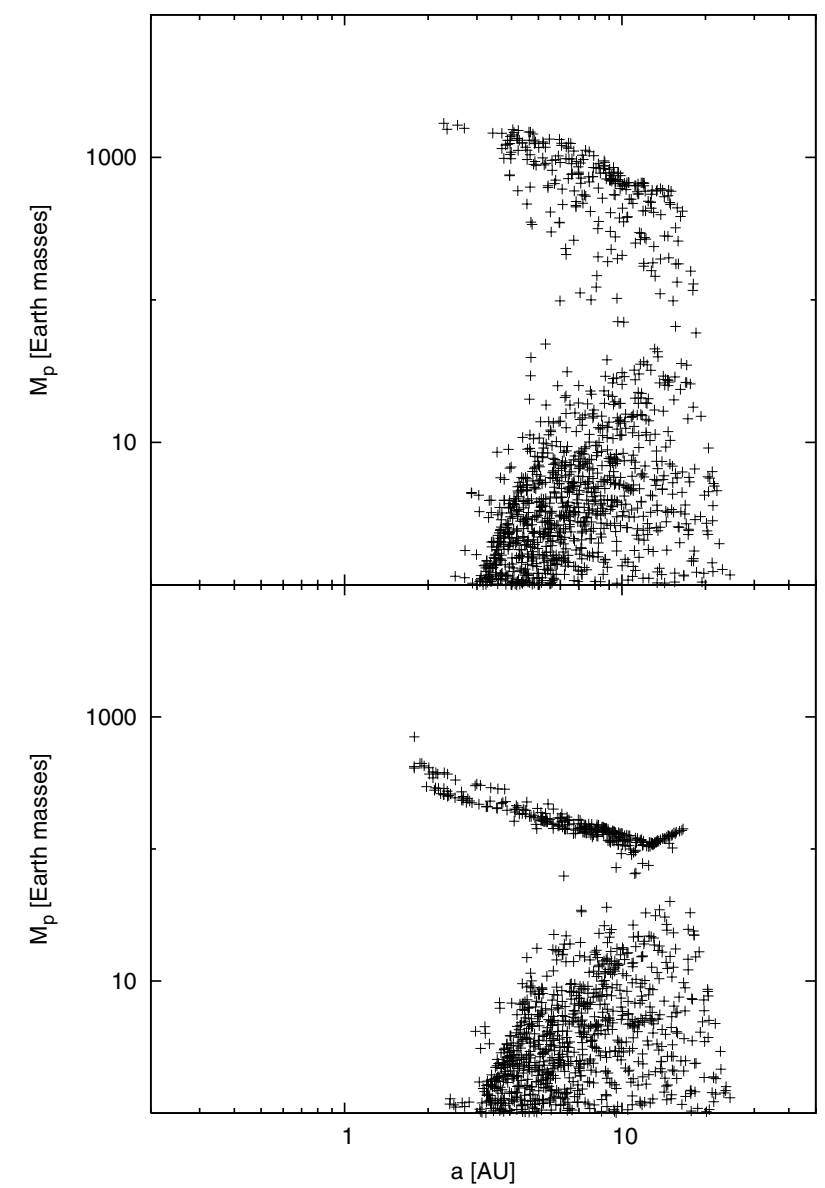

Fig. 3. Effect of planet growth after gap opening on the theoretically predicted distribution of orbital sizes and masses of planets around a star with mass of $1 M_{\odot}$. The upper panel shows the results for planets that undergo type II migration and growth after gap opening. The lower one shows results for planets that undergo type II migration, but not grow after gap opening. The solid material is water ice.

and decreases to $\sim 400 M_{\oplus}$ at $17 \mathrm{AU}$. The increase in the masses of the planets, after gap opening tends to be anticorrelated with the semimajor axes of their orbits. We explain this fact as follows. The rate at which a giant planet accretes gas at this stage of its evolution is determined by the accretion rate in the disk, and as such does not strongly depend on the distance from the star. At the same time the protoplanetary cores at smaller distances grow on shorter timescales. There are two reasons for this. First, the accretion rate of planetesimals onto the core is proportional to Keplerian frequency; second, the surface density of the nascent planetesimal swarm tends to be larger closer to the star. Consequently, protoplanets at smaller orbits are able to start accreting significant amounts of gas for a longer time before dispersion of the disk. Additionally, the accretion rate in the disk, which determines the accretion rate of gas onto the planet, is larger in this earlier epoch. As a result, the maximum size of the planet is everywhere a decreasing function of the distance from the star, even in those regions where the relation was the opposite at the moment of gap opening.

Next, we investigate how the relation between the mass of the planet and semimajor axes of its orbit depends on the sort of material solids consist of. For this purpose we performed similar simulations to those described above, but with solids made of high temperature silicates instead of water ice. For the evaporation temperature of silicates we adopted a value of $1350 \mathrm{~K}$,

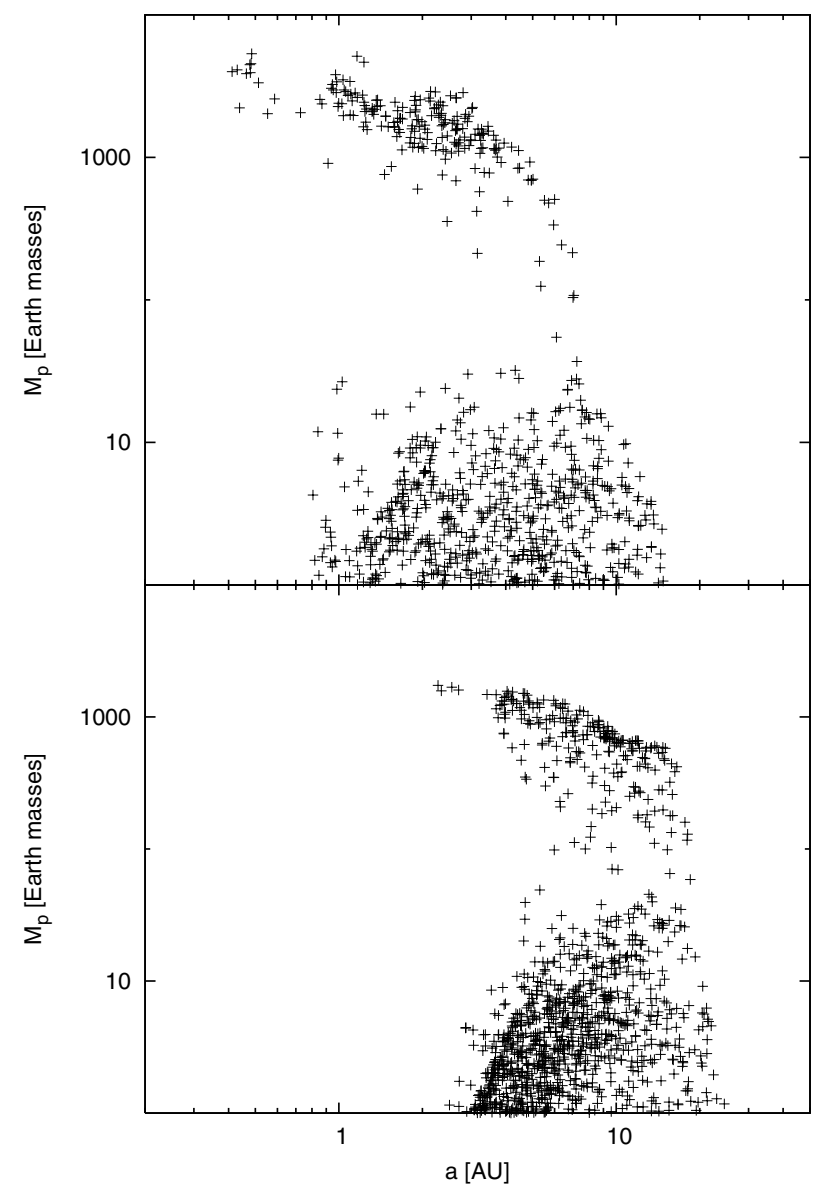

Fig. 4. Effect of different kinds of material that the solids in the protoplanetary disk consist of on the theoretically predicted distribution of orbital sizes and masses of planets around a star with a mass of $1 M_{\odot}$. The upper panel shows the results from models in which solids are composed of silicates, the lower one - from water ice. In both cases the planets undergo type II migration and grow after gap opening.

and for their bulk density $3.3 \mathrm{~g} \mathrm{~cm}^{-3}$. The upper panel of Fig. 4 presents the distribution of planets resulting from these calculations. Because the solids can survive at higher temperatures than ice, the giant planets in that case can form closer to the star. Because the rate of migration does not depend on the composition of solids, their final orbits can also be smaller. At the same time, the distribution of the final surface densities of the planetesimal swarms, as a function of the distance from the star, seems to be a natural extension of this distribution in the case of water ice solids. As a result, the relation of $a-M_{\mathrm{p}}$ for planets with silicate cores at orbits smaller then $6 \mathrm{AU}$ is just an extension to smaller values of $a$ of the same relation for planet with water ice cores.

Consequently, in our models the most massive planets (with $M_{\mathrm{p}} \sim 5000 M_{\oplus}$ ) tend to be located closest to the star at orbits with semimajor axes $\sim 0.5 \mathrm{AU}$. On the other hand, planets with silicate cores at orbits larger than 6 AU tend to have smaller masses than their ice core counterparts. The reason is that at these distances the time during which the core of the protoplanet only accretes solid material becomes a significant fraction of the whole time of its formation. The rate of the accretion of planetesimals onto the core with the same mass, but composed of more dense material, is lower due to its smaller physical dimensions. As a result, planets with silicate cores start their gas accretion later and grow to smaller masses before the dispersion of the 


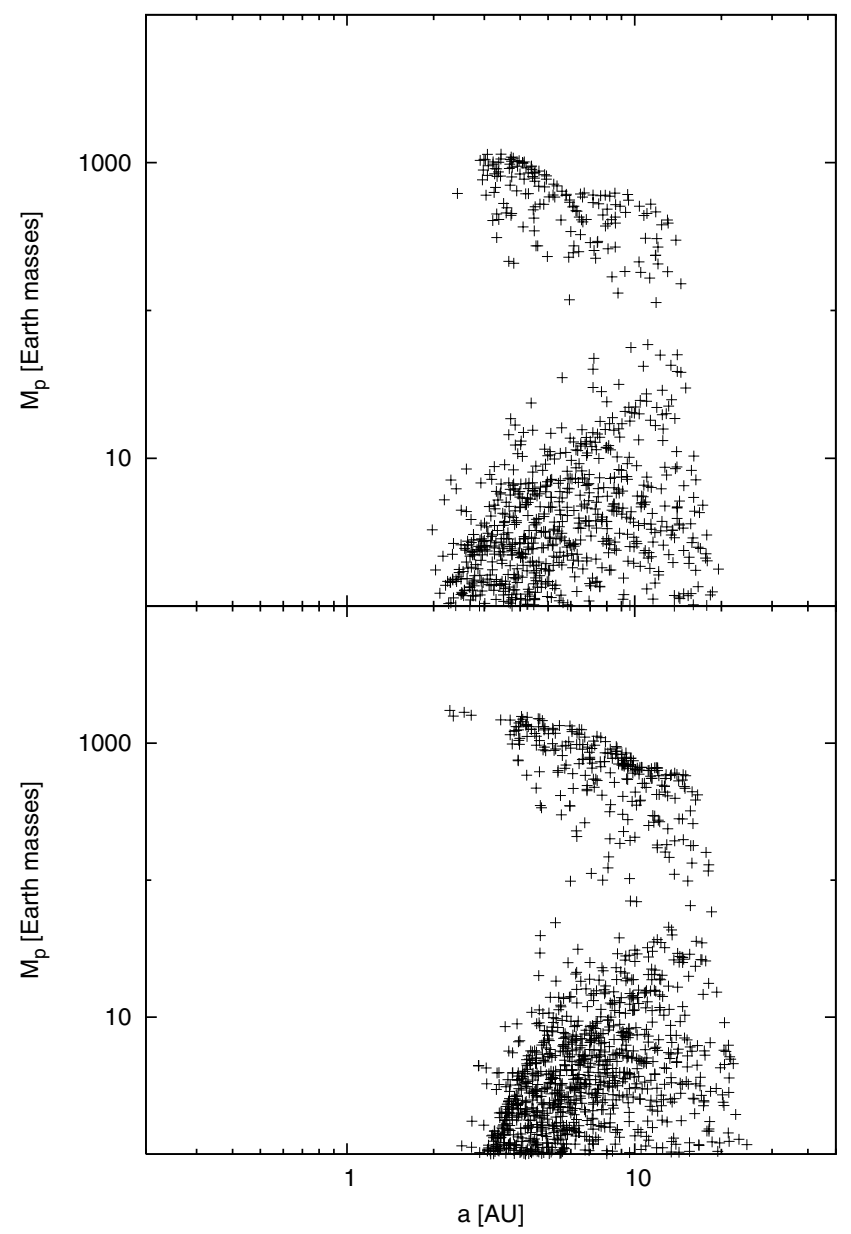

Fig. 5. The distribution of theoretically predicted semimajor axes and masses of planets around stars with masses of $1 M_{\odot}$ (lower panel) and $0.5 M_{\odot}$ (upper panel). In both cases the planets undergo type II migration and grow after gap opening.

disk. At distances larger than $\sim 8 \mathrm{AU}$, we do not obtain any giant planets with silicate cores.

Finally, we investigated the influence of the central star mass on the relation between masses and orbit sizes of giant planets. In Fig. 5 we present the results for two values of the stellar mass: 0.5 and $1 M_{\odot}$. The planets around less massive stars also tend to be less massive and have slightly smaller orbits. The first fact is explained by the lower accretion rates in these disks, as these rates also determine the maximum rate at which the planet can accumulate gas. The tendency of giant planets to form around less massive stars at smaller radii is the result of (a) a more effective radial redistribution of solids in the disk prior to the formation of planetesimals and, as a result the higher surface density of the planetesimal swarm, (b) a lower value of the minimal surface density for the formation of a giant planet around less massive stars at orbits smaller than $\sim 10$ AU (see Kornet et al. 2006).

\section{Conclusions}

We have presented the results of simulations describing the formation of giant planets. Our model include all important phases of the core-accretion scenario, beginning from the formation of the planetesimal swarm from smaller solids, until the gap opening in the disk and subsequent planet migration. We presented the distribution of $a-M_{\mathrm{p}}$ for planets resulting from our models for a set of different initial masses and sizes of the protoplanetary disk and for the place of planet formation. However, our results cannot be compared in a simple way to data for extrasolar planets, because of the lack of knowledge of the distribution of the initial parameters of protoplanetary disks that occur in nature. Instead our aim was to characterize the relationship between the final orbital radii and masses of planets that are in general possible to obtain and to check how the resulting $a-M_{\mathrm{p}}$ distribution changes when including different physical processes such as migration and gas accretion by the planet after gap opening. We also investigated whether the distribution of giant planets is mainly determined by the parameters of the gaseous disk or the distribution of the planetesimal swarm.

We have shown that, if we do not include the effects of migration nor accretion of gas by the planet after gap opening, the $a-M_{\mathrm{p}}$ distribution for giant planets is mainly determined by the parameters of the gaseous disk. In that case one is able to read the information about the dependence of the scale height of the gaseous disk on the distance from the star from the distribution of giant planets. The minimal and maximal distance from the star gives information about the surface density of planetesimal swarm at those places.

By including migration in our models, reconstructing the the properties of the gaseous disk from the distribution of giant planets becomes more difficult. The distance that the planet can move inward depends not only on the gaseous environment but also on the time between gap opening and dispersion of the disk. But the first of these two moments is determined by the surface density of planetesimal swarm. This, on the other hand, is not related in a simple way to the local parameters of the gaseous disk. Our simulations show that the simple reconstruction of the scale height of the gaseous disk is possible only from distribution of giant planets at greater distances than $\sim 8 \mathrm{AU}$, as planets at such large orbits did not significantly move away from their original places.

If we include in our models that the planet can still grow in mass after gap opening, it is even more difficult, if at all possible, to derive conclusions about the gaseous disk (its temperature, scale height, etc.) based on the distribution of giant planets. This is because the rate of this additional accretion onto the planet depends mainly on the accretion rate in the disk, while the time frame in which this accretion can occur is determined by the surface density of the planetesimal swarm. The effect of the additional growth of the planet can even lead to reversion of the correlation between the masses of the planets and semimajor axes of their orbits.

We also checked how the mass of the central star influences the distribution of planets. The main result is that in disks around less massive stars, giant planets at the given location tend to be less massive. At the same time, the giant planets with a given mass tend to form closer to the less massive stars.

Our models can be seen as an extension of previous calculations performed by Ida \& Lin (2004a) and Alibert et al. (2005). In comparison, the surface density of the planetesimal swarms in our models are computed self-consistently. This additional factor makes it much more difficult to predict the structure of the gaseous disk from the distribution of giant planets much more difficult. Nevertheless it does seem necessary. Whenever we performed the similar calculations as described above, but with the assumption that the surface density of the planetesimal swarm is always a constant fraction of the surface density of gas, we were not able to perceive any giant planets. This seems contradict the results of those two papers. A possible reason for this different result may be the difference in the underlying models of the gaseous disk. However, we think that the main factors are 
the different times for the dispersion of the gaseous disk (3 Myr vs. $10 \mathrm{Myr}$ ) and the different sizes of solids at time 0 (small grains vs. planetesimal sizes). Our models show that the time needed to reach planetesimal sizes can be as long $1 \mathrm{Myr}$ and is not negligible.

Our results also seem to contradic observations that show that more massive planets tend to be located farther away from the host star. This correlation is reproduced better by the calculations of Ida \& Lin (2004a), who conclude that maximal mass of the planets after including type II migration does not strongly depend on the size of the orbit. On the other hand, in the results from Alibert et al. (2005) the correlation is identical to ours, namely the more massive planets tend to be closer to the star. This difference can have two sources. First, Ida \& Lin (2004a) neglect the accretion of gas onto the planet, after it opens a gap in the disk. The second reason, which we think is more important, can again be the difference in the structure of the gaseous disk. In our calculations ratio $(H / r)$ is mainly a decreasing function of the radius in the inner parts of the disk, while it is an increasing function in the disk model of Ida \& Lin (2004a). Because this ratio determines the mass of the planet which in turn is able to open a gap in the disk, then it can play a big role in determining the properties of the set of giant planets.

Acknowledgements. This project was supported by the German Research Foundation (DFG) through the Emmy Noether grant WO 857/2-1 and the European Community's Human Potential Programme trough the contract HPRN-CT-2002-00308, PLANETS. KK acknowledge the support from the grant No. 1 P03D 02626 from the Polish Ministry of Science.

\section{References}

Alibert, Y., Mordasini, C., Benz, W., \& Winisdoerffer, C. 2005, A\&A, 434, 343 Bodenheimer, P., Hubickyj, O., \& Lissauer, J. J. 2000, Icarus, 143, 2 Boss, A. P. 2002, ApJ, 576, 462

Bryden, G., Lin, D. N. C., \& Ida, S. 2000, ApJ, 544, 481

Frank, J., King, A., \& Raine, D. 1992, Accretion Power in Astrophysics (Cambridge University Press)

Haisch, K. E., Lada, E. A., \& Lada, C. J. 2001, ApJ, 553, L153

Hubickyj, O. 2001, private communication

Ida, S., \& Lin, D. N. C. 2004a, ApJ, 604, 388

Ida, S., \& Lin, D. N. C. 2004b, ApJ, 616, 567

Ikoma, M., Nakazawa, K., \& Emori, H. 2000, ApJ, 537, 1013

Kornet, K., Stepinski, T. F., \& Różyczka, M. 2001, A\&A, 378, 180

Kornet, K., Bodenheimer, P., \& Różyczka, M. 2002, A\&A, 396, 977

Kornet, K., Bodenheimer, P., Różyczka, M., \& Stepinski, T. F. 2005, A\&A, 430, 1133

Kornet, K., Wolf, S., \& Różyczka 2006, A\&A, in press

Lin, D. N. C., \& Papaloizou, J. 1986, ApJ, 309, 846

Lin, D. N. C., Bodenheimer, P., \& Richardson, D. C. 1996, Nature, 380, 606

Marcy, G. W., Butler, R. P., Vogt, S. S., et al. 2005, ApJ, 619, 570

Mayor, M., Udry, S., Naef, D., et al. 2004, A\&A, 415, 391

Nelson, R. P., \& Papaloizou, J. C. B. 2004, MNRAS, 350, 849

Papaloizou, J. C. B., \& Terquem, C. 1999, ApJ, 521, 823

Podolak, M., Pollack, J. B., \& Reynolds, R. T. 1988, Icarus, 73, 163

Pollack, J. B., Hubickyj, O., Bodenheimer, P., et al. 1996, Icarus, 124, 62

Shakura, N. I., \& Sunyaev, R. A. 1973, A\&A, 24, 337

Stepinski, T. F. 1998, Icarus, 132, 100

Stepinski, T. F., \& Valageas, P. 1997, A\&A, 319, 1007

Veras, D., \& Armitage, P. J. 2004, MNRAS, 347, 613

Ward, W. R. 1997, ApJ, 482, L211

Weidenschilling, S. J. 2003, in Lunar and Planetary Science Conference \#34, abstract \#1707

Weidenschilling, S. J., \& Cuzzi, J. N. 1993, in Protostars and Planets III, ed. E. Levy, \& J. Lunine (Tuscon: Univ. of Arizona Press), 1031 